\title{
Mirror-orientation noise in a Fabry-Perot interferometer gravitational wave detector
}

\author{
Seiji Kawamura and Michael E. Zucker
}

\begin{abstract}
The influence of angular mirror-orientation errors on the length of a Fabry-Perot resonator is analyzed geometrically. Under conditions in which dominant errors are static or vary slowly over time, the analysis permits a simple prediction of the spectrum of short-term cavity length fluctuations resulting from mirror-orientation noise. The resulting model is applicable to the design of mirror control systems for the Laser Interferometer Gravitational-Wave Observatory, which will monitor separations between mirrored surfaces of suspended inertial test bodies as a way to measure astrophysical gravitational radiation. The analysis is verified by measuring the response of the Laser Interferometer GravitationalWave Observatory's 40-m interferometer test-bed to the rotation of its mirrors.

Key words: Fabry-Perot, cavity, gravitational wave, interferometer, alignment.
\end{abstract}

\section{Introduction}

The Laser Interferometer Gravitational-Wave Observatory (LIGO) will employ Fabry-Perot optical cavities as a way to detect and measure small changes in separation, having characteristic durations of a few milliseconds and magnitudes of the order of $10^{-18} \mathrm{~m}$, between inertial gravitational test bodies separated by orthogonal $4-\mathrm{km}$ baselines. ${ }^{1,2}$ The four test bodies, made of fused quartz, will be polished and coated so that two resonant Fabry-Perot optical cavities are formed. Laser light will be split by a beam splitter and made to resonate in each of the two orthogonal cavities; the resonant fields will be extracted and interfered to measure the difference between their phases, which depend sensitively on the mirror separations. For the test bodies to be isolated from external forces, they are to be suspended as pendula whose natural periods, of the order of $1 \mathrm{~s}$, are significantly longer than characteristic signal time scales. Band-limited control systems will damp their rotational rigid-body normal modes, which also will have periods of the order of $1 \mathrm{~s}$, so that optical alignment of the resonators is maintained. The required displacement sensitivity places a direct limit on the permissible linear momentum imparted to test

The authors are with the Laser Interferometer GravitationalWave Observatory Project, California Institute of Technology, Pasadena, California 91125.

Received 11 January 1993; revised manuscript received 12 October 1993.

0003-6935/94/183912-07\$06.00/0.

(1994 Optical Society of America. bodies by seismic noise, thermal fluctuations, and side effects of the control systems.

Excess noise from angular fluctuations of each mirror, arising from external torques, also influences optical cavity length. A simple geometric model predicting the apparent cavity length change caused by test-body rotation was developed and evaluated on the LIGO 40-m interferometer test-bed. The model accurately predicts the interferometer's response to experimental probe torques applied to its test bodies. Applying the model to predict the effect of residual torques from the interferometer's mirror angle control systems indicated that such noise limited the interferometer's sensitivity at frequencies below 700 $\mathrm{Hz}$. New lower-noise control systems were developed and implemented, resulting in a substantial improvement in displacement sensitivity.

\section{Optical Cavity Length}

The round-trip optical phase for the resonator's $\mathrm{TEM}_{00}$ mode, which is measured and interpreted as apparent mirror displacement, depends on the optical length $l$, which is the length of the line segment that lies perpendicular to both mirror surfaces (i.e., the optic axis). Our objective is to quantify the relationship between the measured quantity $l$ and the desired inertial length $L$, the separation between the test bodies' centers of mass, as each body rotates in response to external torques. We employ the orthogonality of small rotations about the two relevant mutually perpendicular axes (altitude and azimuth) to treat their influences on $l$ independently; thus, in what follows, the normals to both mirrors and the 
line joining the test bodies can be assumed to lie in a common plane. We also assume that no net forces are applied (i.e., $L$ is constant).

The optical length of a Fabry-Perot cavity depends on the deviation angles $\theta_{1}$ and $\theta_{2}$, defined as shown in Fig. 1. These angles are both equal to zero when the optical axis coincides with the line joining the centers of mass of the test bodies. To second order in $\theta_{1}$ and $\theta_{2}$, which are presumed to be small, the optical length of the cavity is given by ${ }^{3}$

$$
l=l_{0}+\alpha \theta_{1}^{2}+\beta \theta_{2}^{2}+\gamma \theta_{1} \theta_{2},
$$

where, for the half-symmetric cavity geometry illustrated, ${ }^{4}$

$$
\begin{aligned}
& \alpha=\frac{1}{2}\left(R+a_{2}-L\right), \\
& \beta=-\frac{\gamma}{2}=\frac{1}{2}\left(R+a_{2}\right) .
\end{aligned}
$$

Here $R$ is the radius of curvature of concave mirror $\mathrm{M}_{2}$ and $l_{0} \equiv L-a_{1}-a_{2}$ is the optical length when $\theta_{1}=$ $\theta_{2}=0$. The time evolution $l(t)$ can be computed from Eq. (1) for arbitrary $\theta_{1}(t)$ and $\theta_{2}(t)$.

For typical cases of experimental interest, $\theta_{1}$ and $\theta_{2}$ will be random processes whose properties are summarized by measured power spectral density functions. We are interested in estimating the power spectrum of the resulting random process $l$ for comparison with expected signals and other noise. Because the power spectrum is the expectation value of the squared modulus of a random-process Fourier transform, ${ }^{5}$ we begin by transforming Eq. (1) into the frequency domain. By the convolution theorem, the transform of Eq. (1) can be written as

$$
\tilde{l}(f)=l_{0} \delta(f)+\alpha \tilde{\theta}_{1} \otimes \tilde{\theta}_{1}(f)+\beta \tilde{\theta}_{2} \otimes \tilde{\theta}_{2}(f)+\gamma \tilde{\theta}_{1} \otimes \tilde{\theta}_{2}(f),
$$

where $\delta(f)$ is the Dirac delta function and the operator $\otimes$ denotes convolution;

$$
\tilde{a} \otimes \tilde{b}(f) \equiv \int_{-\infty}^{\infty} \tilde{a}\left(f^{\prime}\right) \tilde{b}\left(f-f^{\prime}\right) \mathrm{d} f^{\prime} .
$$

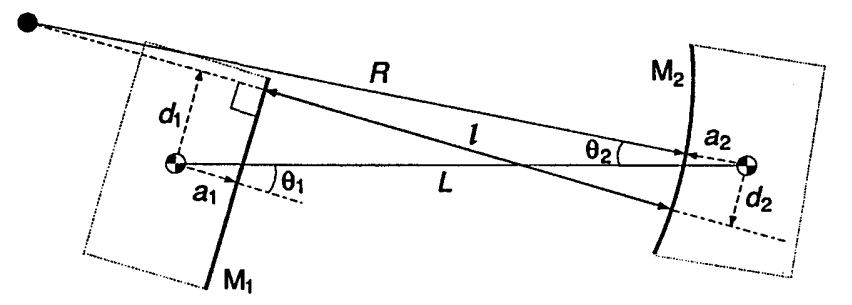

center of curvature $\left(\mathrm{M}_{2}\right)$
center of mass

Fig. 1. Geometry of a half-symmetric Fabry-Perot cavity consisting of flat $\left(\mathrm{M}_{1}\right)$ and concave $\left(\mathrm{M}_{2}\right)$ test-body mirrors.
We will later replace each angle fluctuation's Fourier transform with the square root of the spectral density of the corresponding random process, and we take the result to be the square root of the spectral density of $l$. For this substitution to be justified the phases of the Fourier components must truly be random, that is, components at different frequencies must not on average be correlated. True random-noise processes such as those considered here will satisfy this criterion. ${ }^{6}$

Although Eq. (3) applies to any Fabry-Perot cavity, the application to gravitational wave detection permits an intuitively appealing simplification. Terrestrial gravitational wave detectors will monitor $l(t)$ only at frequencies above a few tens of hertz, whereas the alignment errors $\theta_{1}$ and $\theta_{2}$ are dominated by static (dc) or slowly varying components (at frequencies below $10 \mathrm{~Hz}$ ). Faster angle fluctuations, at frequencies within the observation band, will be considerably smaller. This spectral character arises from longterm thermal drift and the increase of seismic vibration with decreasing frequency, combined with the low-pass filtering action of the suspension. In such a situation we may write

$$
\theta_{\nu}(t)=\bar{\theta}_{\nu}+\varepsilon_{\nu}(t)
$$

where $\left|\varepsilon_{v}(t)\right| \ll\left|\bar{\theta}_{v}\right|$,

$$
\bar{\theta}_{v} \equiv \frac{1}{T} \int_{-T / 2}^{T / 2} \theta_{v}(t) \mathrm{d} t
$$

is the average taken over some long interval $T$, and $\nu=1$ or $v=2$ for mirror $M_{1}$ or $M_{2}$, respectively. To leading order in $\varepsilon_{v} / \bar{\theta}_{v}$ we find

$$
l(t) \simeq l_{0}+\frac{1}{2} \bar{d}_{1}\left[\bar{\theta}_{1}+2 \varepsilon_{1}(t)\right]+\frac{1}{2} \bar{d}_{2}\left[\bar{\theta}_{2}+2 \varepsilon_{2}(t)\right],
$$

where the quantities

$$
\begin{aligned}
& d_{1} \equiv 2 \alpha \theta_{1}-2 \beta \theta_{2}, \\
& d_{2} \equiv 2 \beta\left(\theta_{2}-\theta_{1}\right)
\end{aligned}
$$

are the moment arms separating the perturbed optic axis (i.e., the position of the resonating beam on each mirror) from each test body's center of rotation (Fig. 1). The Fourier transform of relation (7) is then simply

$$
\tilde{l}(f) \approx \bar{d}_{1} \tilde{\varepsilon}_{1}(f)+\bar{d}_{2} \tilde{\varepsilon}_{2}(f) \quad(f \neq 0),
$$

agreeing with the intuitive notion that the apparent displacement of the portion of the mirror at the beam location is given by the angle fluctuation multiplied by the moment arm.

Relation (9) can be generalized if we let $\bar{\theta}_{\nu}$ be a large (in the mean-square sense), slowly varying, rather than static, misalignment. If the dominant misalignment is confined to a small region of the spectrum 
$|f|<|w|$, we can write

$$
\tilde{\theta}_{\nu}(f)=\tilde{\theta}_{\nu}{ }^{w}(f<w)+\tilde{\varepsilon}_{\nu}(f>w),
$$

where

$$
\int_{-w}^{w}\left|\tilde{\theta}_{\nu}{ }^{w}(f)\right|^{2} \mathrm{~d} f \gg \int_{-\infty}^{\infty}\left|\tilde{\varepsilon}_{\nu}(f)\right|^{2} \mathrm{~d} f,
$$

i.e., the low-frequency portion carries substantially more spectral power than the high-frequency portion. We then find that the analogous expression to relation (9), again to leading order, is

$$
\begin{aligned}
\tilde{l}(f) \simeq & \int_{-w}^{w} \tilde{d}_{1}\left(f^{\prime}\right) \tilde{\varepsilon}_{1}\left(f-f^{\prime}\right) \mathrm{d} f^{\prime} \\
& +\int_{-w}^{w} \tilde{d}_{2}\left(f^{\prime}\right) \tilde{\varepsilon}_{2}\left(f-f^{\prime}\right) \mathrm{d} f^{\prime}(f>2 w) .
\end{aligned}
$$

One can also derive this result by restricting the range of the convolution integrals in Eq. (3) to frequencies in which at least one parent spectrum carries significant power.

For a static misalignment, $\tilde{d}_{\nu}(f)=\bar{d}_{\nu} \delta(f)$, we recover relation (9), and a mirror angle fluctuation at frequency $f_{0}$ linearly induces a Fourier component of optical length at the same frequency. This can be shown to give an adequate prediction of the optical length spectrum, without resorting to the more general form of relation (12), if $\bar{d}_{v} \gg d_{v}^{\text {rms }}$, where

$$
\begin{aligned}
\left(d_{\nu}^{\mathrm{rms}}\right)^{2} & \equiv \frac{1}{T} \int_{0}^{T}\left[d_{\nu}(t)-\bar{d}_{\nu}\right]^{2} \mathrm{~d} t \\
& =\int_{-w}^{w}\left|\tilde{d}_{\nu}(f)\right|^{2}[1-\delta(f)] \mathrm{d} f
\end{aligned}
$$

is the mean-square deviation of the optic axis (excluding the static offset).

In contrast, if $\bar{d}_{v} \lesssim 2 d_{v}{ }^{\text {rms }}$, the optical path-length spectrum will not be linearly related to the angle spectrum and will contain frequency-shifted sideband products. Nevertheless, in situations of interest, the high-frequency mirror angle spectra $\tilde{\varepsilon}_{v}(f)$ are often smooth and nearly constant over a band of frequencies $\Delta f \geq 2 w$. Such spectra arise from electronic noise in the mirror control system or from thermal fluctuations in the suspension, for example. We can then derive the approximation

$$
\begin{aligned}
|\tilde{l}(f)|^{2} \approx & {\left[\bar{d}_{1}^{2}+\left(2 d_{1}^{\mathrm{rms}}\right)^{2}\right]\left|\tilde{\varepsilon}_{1}(f)\right|^{2} } \\
& +\left[\bar{d}_{2}{ }^{2}+\left(2 d_{2}^{\mathrm{rms}}\right)^{2}\right]\left|\tilde{\varepsilon}_{2}(f)\right|^{2} \quad(f>2 w) .
\end{aligned}
$$

Under the above conditions, we may replace the squared Fourier transforms with the power spectral densities $S_{\varepsilon_{1}}(f)$ and $S_{\varepsilon_{2}}(f)$ of random processes $\varepsilon_{1}$ and $\varepsilon_{2}$ to obtain

$$
\begin{aligned}
S_{l}(f) \approx & {\left[\bar{d}_{1}^{2}+\left(2 d_{1}^{\mathrm{rms}}\right)^{2}\right] S_{\varepsilon_{1}}(f) } \\
& +\left[\bar{d}_{2}{ }^{2}+\left(2 d_{2}^{\mathrm{rms}}\right)^{2}\right] S_{\varepsilon_{2}}(f) \quad(f>2 w),
\end{aligned}
$$

where $S_{l}(f) \equiv \lim _{T \rightarrow \infty} 2|\tilde{l}(f)|^{2} / T$ is the power spectral density of induced cavity length fluctuation.

In the paragraphs above we treat static and rms errors as separate coupling parameters for purely practical reasons, related to our intended application. The specialization of $d_{\nu}$ rms to specifically exclude dc is motivated by the qualitative difference in the origins and methods for dealing with fluctuations that occur on time scales much longer than gravitational wave measurements, as opposed to those that occur on comparable or shorter time scales. Long-term misalignments in gravitational wave interferometers result from thermal, tidal, or relaxation effects, and they can be reduced to an acceptable level by periodic readjustment. Shorter-term misalignments, generally driven by seismic noise, are only reduced to a finite extent by action of the mirror control system; the residual rms seismic excitation thus constitutes a practical lower limit to the coupling between angle and length.

\section{Experimental Tests}

This model was tested on the LIGO 40-m interferometer. ${ }^{7}$ Briefly, this instrument comprises two orthogonal $40-\mathrm{m}$ cavities whose $1.5-\mathrm{kg}$ test bodies are suspended by wires so they are essentially free to translate in a horizontal plane and to rotate about axes perpendicular to the laser beam (Fig. 2). The geometrical parameters of each cavity are $R=62 \mathrm{~m}$, $L=40 \mathrm{~m}$, and $a_{1}=a_{2}=6 \mathrm{~cm}$ (Fig. 1 ).

The pendulum mode and both rotational normal modes of the suspended bodies have eigenfrequencies near $1 \mathrm{~Hz}$. Each is controlled in azimuth $(\phi)$ and elevation $(\theta)$ by a control system that derives appropriate feedback signals from an optical lever sensor and applies magnetic corrective torques to the suspension. Manual offsets can be introduced and trimmed as a way to adjust and optimize (or degrade) the alignment of the two cavities.

The control electronics are provided with summing nodes, through which one may introduce probe signals to measure the transfer characteristics for various degrees of freedom. We calibrate the induced rotations by measuring the motion of the optical lever reflections. The interferometer's differential displacement output signal, which represents the difference between the cavity optical lengths, is monitored and itself periodically calibrated against a known test force applied electromagnetically to one test body. The differential displacement induced by the probe signal reflects the change in the length $l(t)$ of the probed cavity. We Fourier analyze this length signal with a digital spectrum analyzer to pick out the components resulting from the probes (while discrimi- 


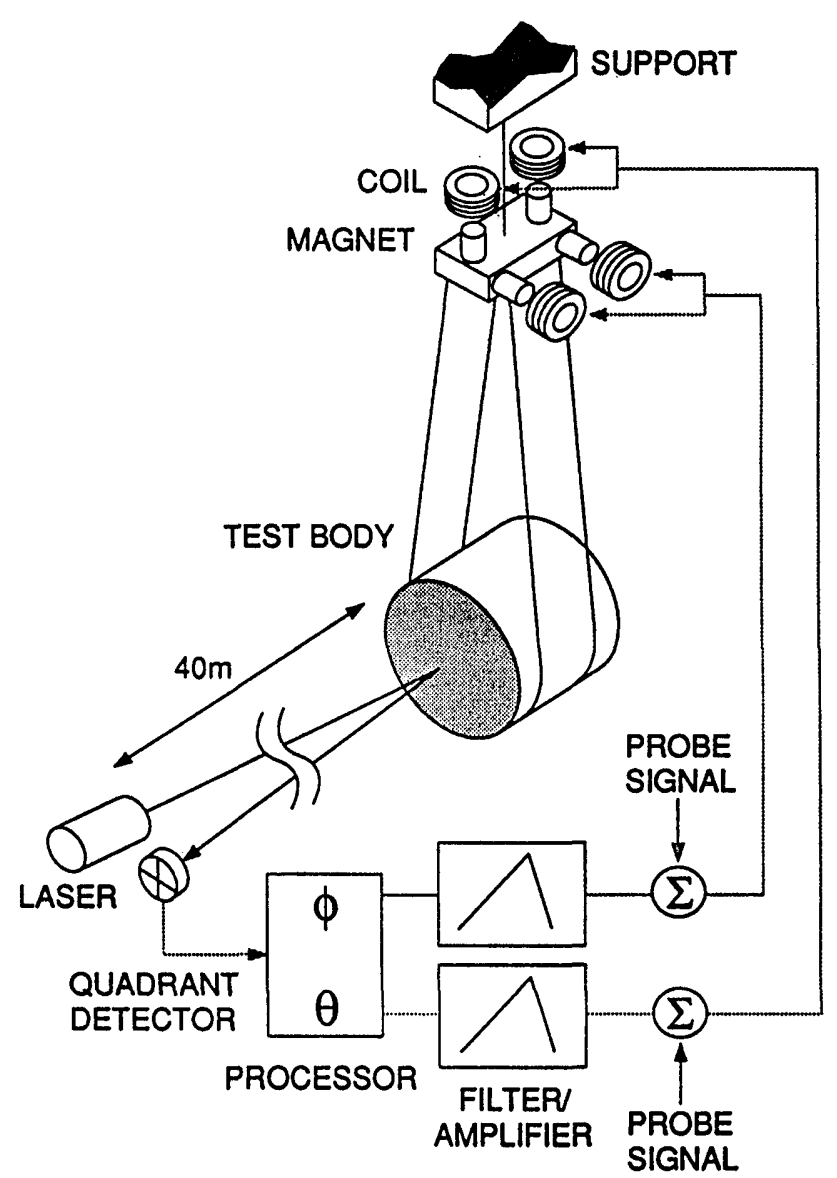

Fig. 2. Suspended test-body and orientation control system used in the LIGO $40-\mathrm{m}$ interferometer. The quadrant photodetector and electronic processor determine angular error signals from the position of an auxiliary laser beam reflected from the mirrored surface of the test body. These signals are filtered and amplified and applied to pairs of electromagnetic coils near the suspension point. The coils interact with permanent magnets (poled oppositely to induce torque) on an intermediate platform, which is suspended by a single wire so that it is free to rotate. The torque developed on this platform is transmitted to the test body below by the suspension wires, inducing rotation of the mirror and closing the feedback loop. Probe torques are introduced by the addition of currents to the feedback signals driving the coils.

nating against other components) and to measure their power spectra.

In the presence of a large static dc misalignment $\bar{d}_{v}$ between the cavity axis and one mirror's center of mass, relation (9) implies that the coupling of that mirror's angle to apparent cavity length will be $\tilde{l}(f) / \tilde{\varepsilon}_{v}(f)=\bar{d}_{\nu}$. To test this prediction, we applied a small sinusoidal probe torque resulting in a vertical oscillation $\varepsilon_{2}(t)=a \cos \left(2 \pi f_{0} t\right)$ with $a=3.5 \times 10^{-9} \mathrm{rad}$ to one mirror $\left(\mathrm{M}_{2}\right.$ in Fig. 1) at $f_{0}=250 \mathrm{~Hz}$. We calibrated the probe amplitude by observing the deflection of an optical lever beam reflected from that mirror. We recorded the interferometer cavity length signal, as the vertical distance $\bar{d}_{2}$ between the cavity axis and the center of that mirror was varied, by adjusting the alignment controls for both mirrors and the direction of the incident laser beam. We moni- tored the true position of the cavity axis by photographing scattered light from the resonating cavity mode against the outline of the mirror. Figure 3 shows the Fourier component of the measured cavity length at $f_{0}$ divided by the induced mirror angle probe amplitude, $\tilde{l}\left(f_{0}\right) / \tilde{\varepsilon}_{2}\left(f_{0}\right)$, versus $\bar{d}_{2}$. Because the exact position of the test body's center of mass was not accurately known, we chose the reference point $\bar{d}_{2}=$ 0 ; the measured slope agrees, within experimental errors, with that predicted by relation (9).

In more realistic situations, the mirror angle spectra will consist of many uncorrelated components, so the cross products of many different frequency pairs will generally be superimposed at each frequency in the convolved result. To investigate this case, we generated a band-limited random-noise test signal to induce random angle fluctuations of $\mathrm{M}_{2}$ principally between 200 and $315 \mathrm{~Hz}$ :

$$
\left|\tilde{\varepsilon}_{2}(f)\right| \approx \begin{cases}0, & f \leq 200 \mathrm{~Hz} \\ K, & 200 \mathrm{~Hz} \leq f \leq 315 \mathrm{~Hz}, \\ 0, & f \geq 315 \mathrm{~Hz}\end{cases}
$$

where $K$ is a constant. The cavity length's spectral density was monitored in this frequency band as a function of $\bar{d}_{2}$. As shown in Fig. 4, the magnitude of the effective transfer function for this random-noise probe signal is approximately proportional to the magnitude of $\bar{d}_{2}$ at large offsets, as in Fig. 3, but it remains essentially constant for very small $\bar{d}_{2}$. To investigate this behavior, we analyzed the light transmitted through $\mathrm{M}_{2}$ with a position-sensing quadrant photodetector to measure low-frequency fluctuation of the cavity axis position $\tilde{d}_{2}\left(f \ll f_{0}\right)$. Integrating this position spectrum over frequency [and excluding dc as in Eq. (13)] gave $d_{2}{ }^{\mathrm{rms}} \approx(0.2 \pm 0.1) \mathrm{mm}$. From Eq. (15) we expect the linear proportionality approximation to fail for $\left|\bar{d}_{2}\right| \lesssim 2 d_{2}{ }^{\mathrm{rms}} \approx(0.4 \pm 0.2) \mathrm{mm}$, essentially the region in which the data shown in Fig. 4 deviate from the linear prediction. Although the method we used to obtain the data in Figs. 3 and 4 can

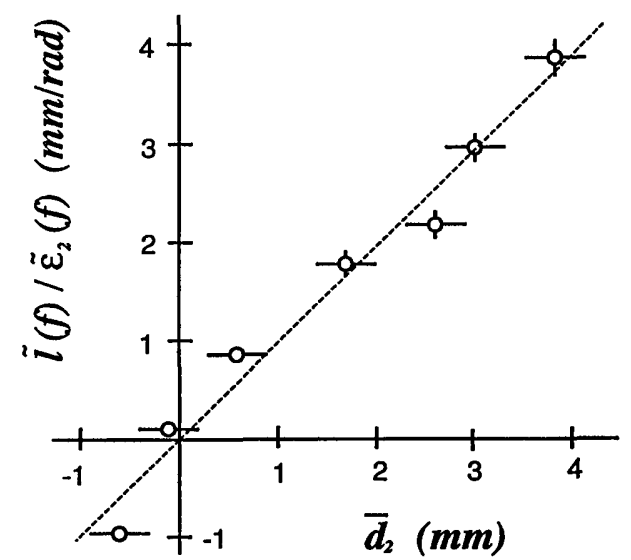

Fig. 3. Linear mirror angle-cavity length coupling coefficient $\tilde{l}\left(f_{0}\right) /$ $\bar{\varepsilon}_{2}\left(f_{0}\right)$ as a function of beam axis position $\bar{d}_{2}$. The dashed curve is the curve $\tilde{l}\left(f_{0}\right) / \tilde{\varepsilon}_{2}\left(f_{0}\right)=\bar{d}_{2}$ predicted by relation (9). The zero for the $\bar{d}_{2}$ axis has been chosen for best fit. 


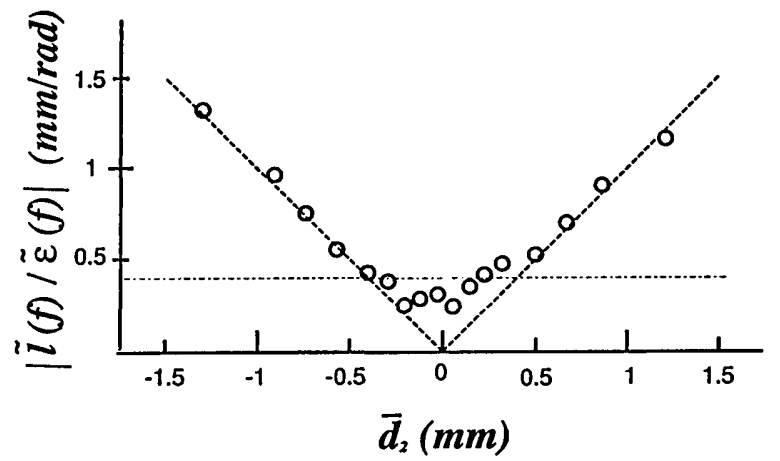

Fig. 4. Interferometer displacement caused by band-limited random mirror angle noise as a function of $\bar{d}_{2}$. The coupling magnitude appears proportional to $\left|\bar{d}_{2}\right|$ down to $\left|\bar{d}_{2}\right| \approx 0.4 \mathrm{~mm}$, below which it is approximately constant. By integrating the spectrum of position fluctuations in the transmitted cavity mode, we found that $d_{2}{ }^{\mathrm{rms}} \approx 0.2 \mathrm{~mm} \pm 0.1 \mathrm{~mm}$ during this experiment, which agrees with the transition from linear behavior predicted by relation (15).

be used for each mirror for the empirical adjustment of all $\bar{d}_{v}$ precisely to zero, the average coupling coefficient will generally reach a nonzero minimum of order $2 d_{v}$ rms.

To test relation (12) quantitatively, we imposed a two-frequency probe signal

$$
\tilde{\theta}_{2}(f)=\theta_{2}{ }^{a} \delta\left(f_{a}\right)+\varepsilon_{2} \delta\left(f_{b}\right)
$$

on $\mathrm{M}_{2}$ with a large low-frequency component $\left(\theta_{2}{ }^{a}=1.6 \times 10^{-6} \mathrm{rad}_{\mathrm{rms}}, f_{a}=10 \mathrm{~Hz}\right)$ and a small high-frequency component $\left(\varepsilon_{2}=5.0 \times 10^{-9} \mathrm{rad}_{\mathrm{rms}}\right.$, $\left.f_{b}=250 \mathrm{~Hz}\right)$. The natural $\tilde{\theta}_{2}(f)$ spectrum was small enough to be dominated completely by these probe components in their respective frequency regimes. The cavity length signal displayed the expected pair of equal-amplitude Fourier components at 260 and 240 $\mathrm{Hz}$, with measured amplitudes of $(3.9 \pm 1.0) \times 10^{-13}$ $\mathrm{m}_{\mathrm{rms}}$; direct substitution of the probe spectrum Eq. (17) into relation (12) would predict

$$
\tilde{l}(f)=3.4 \times 10^{-13} \mathrm{~m}_{\mathrm{rms}} \times\left[\delta\left(f_{b}+f_{a}\right)+\delta\left(f_{b}-f_{a}\right)\right],
$$

which agrees within measurement errors.

The $f_{a}=10 \mathrm{~Hz}$ component of the probe rotation was then removed, and the $250-\mathrm{Hz}$ test signal was reduced in amplitude to $\varepsilon_{2}=1.5 \times 10^{-9} \mathrm{rad}_{\mathrm{rms}}$. An analysis of the cavity length spectrum then revealed natural sidebands, symmetric around the $250-\mathrm{Hz}$ probe. The vertical position of the cavity axis at $\mathrm{M}_{2}$ was simultaneously measured by the transmittedbeam quadrant photodetector. As shown in Fig. 5, the detailed spectral shape found for the cavity length signal matches the spectrum of the cavity axis position, upshifted by $250 \mathrm{~Hz}$ and with an amplitude close to that predicted by relation (12). In effect, the monochromatic $250-\mathrm{Hz}$ probe has acted as a virtual delta function in the convolution integral, picking out of the convolution an upshifted replica of the residual low-frequency beam offset spectrum.
Application Examples

The following examples illustrate the use of relation (15) as a way to understand the effect of orientation fluctuations.

\section{0-m Interferometer}

We applied our analysis in 1991 to the 40-m interferometer to analyze its noise spectrum and to achieve a significant improvement in its sensitivity. The transfer functions from mirror torque control signals to mirror angles, and the residual noise in these control signals were measured and combined with estimates of the displacement between each cavity mirror's center of mass and the optical axis of that cavity, thereby forming an estimated noise contribution from each angular degree of freedom to the total displacement noise. These estimates indicated that mirror-orientation noise was a significant component of the observed displacement spectrum. ${ }^{8}$ We confirmed the conclusion by demonstrating that improved coincidence between optical and inertial axes (achieved by iterative realignment) or reduced highfrequency angle noise (achieved by reducing control system gain) could reduce interferometer noise over a broad spectral band, principally between 50 and $700 \mathrm{~Hz}$.

Although high-frequency angle fluctuations can arise from many phenomena, the dominant source in this case was found to be excess noise in the mirrororientation control systems, resulting from a combination of direction and intensity fluctuation in the optical lever laser beams, electronic noise in control electronics, and inadequate filtering outside the control band. Improved feedback electronics were devel-

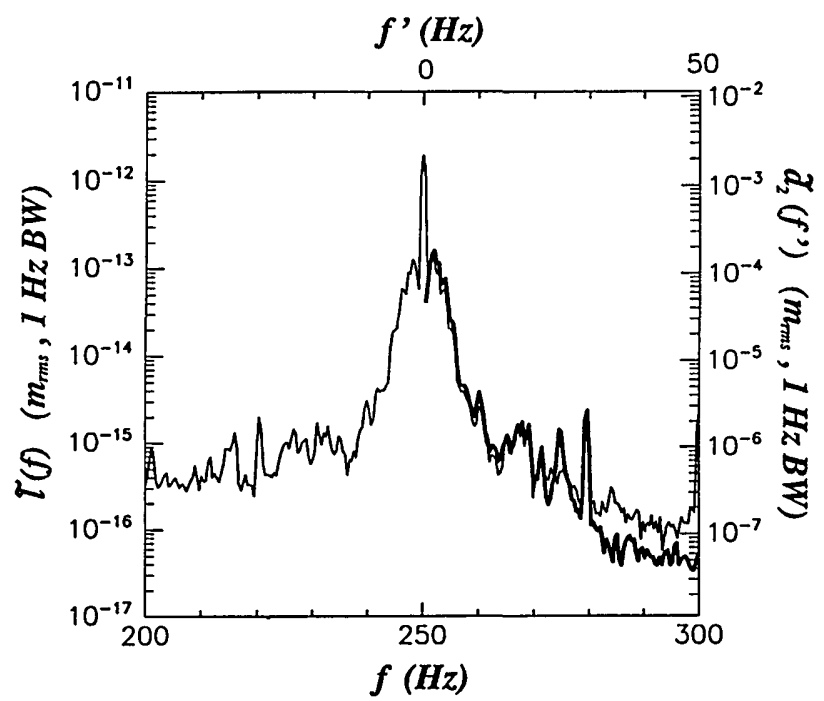

Fig. 5. Spectrum of low-frequency beam axis position fluctuations (thick curve, upper frequency scale and right-hand magnitude scale) replicated as upper and lower sidebands of an artificially induced $250-\mathrm{Hz}$ probe angle fluctuation in the interferometer displacement spectrum (thin line, lower and left-hand scales). We chose the relative scales in accord with relation (12) by using the known $1.5 \times 10^{-9} \mathrm{rad}_{\mathrm{rms}}$ amplitude of the $250-\mathrm{Hz}$ probe. Both spectra are normalized to bundwidth $\mathrm{BW}=1 \mathrm{~Hz}$. 
oped with lower unity gain frequency, increased filter attenuation, and lower intrinsic electronic noise. At frequencies near $100 \mathrm{~Hz}$, the improved controllers would contribute a factor of $10^{5}$ less noise than the systems they replaced for a given offset between cavity optical and inertial axes. This change reduced the interferometer displacement noise by a significant factor at frequencies between 50 and $700 \mathrm{~Hz}$, leaving the sensitivity limited by other noise mechanisms except at narrow mechanical resonances (Fig. 6).

\section{LIGO Interferometer}

Specifications for LIGO test-body orientation controls can also be derived by application of the model. Initial LIGO interferometers are expected to achieve total displacement noise spectral densities below $10^{-19}$ $\mathrm{m} / \sqrt{\mathrm{Hz}}$ at frequencies near $100 \mathrm{~Hz}^{1}$ To meet this goal, relation (15) permits angular fluctuations no larger than

$$
S_{\theta_{v}}{ }^{1 / 2}(f) \lesssim 10^{-17} \frac{\mathrm{rad}}{\sqrt{\mathrm{Hz}}}
$$

for each angular degree of freedom of the four cavity mirrors at frequencies $f \sim 100 \mathrm{~Hz}$, assuming that the projected $d_{\nu}{ }^{\mathrm{rms}} \sim 0.5 \mathrm{~mm}$ is achieved at remote LIGO sites. ${ }^{9}$ Here we have omitted the additional linear contribution caused by nonzero $\bar{d}_{\nu}$, because we can empirically trim it to zero by monitoring the response of each mirror to probe torques; see Figs. 3 and 4.

The improved 40-m interferometer mirror control systems described above induce approximately $5 \times$

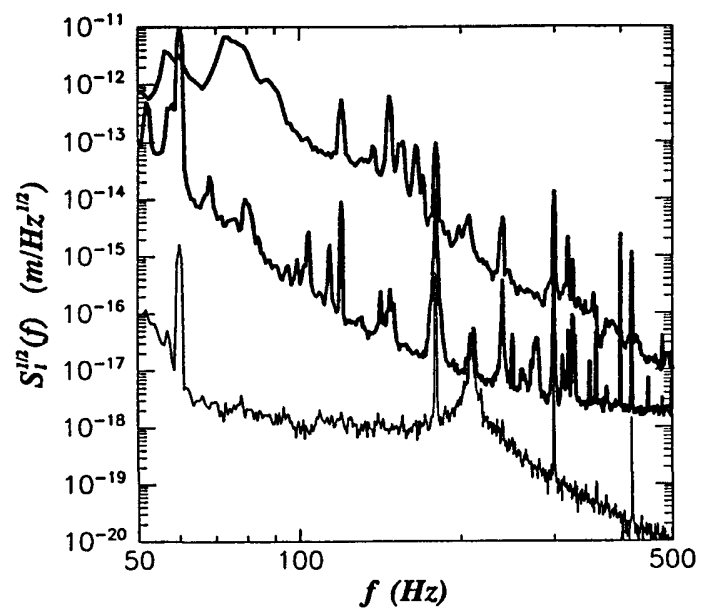

Fig. 6. Spectral density of apparent cavity mirror displacement in the $40-\mathrm{m}$ interferometer with the original orientation control system (upper thick curve) and after installing the new orientation feedback electronics (middle thick curve). The lower, thin curve depicts the estimated noise contributed by rotation of one of the test bodies about its horizontal axis. We formed this estimate by assuming that assumed $\bar{d}_{2} \approx 1 \mathrm{~mm}$ in relation (15) to project the typical influence of the new control circuit's residual electronic noise. The peak at $212 \mathrm{~Hz}$ is a resonance of the wire suspension system, where the coupling of external torque to the mirror is locally enhanced.
$10^{-16} \mathrm{rad} / \sqrt{\mathrm{Hz}}$ angle fluctuations near $100 \mathrm{~Hz}$, primarily as a result of electronic noise in their active filters. Substitution of passive or lower-noise active filtering would give a reduction in noise by a factor of 30 or more at $100 \mathrm{~Hz}$. In addition, the remote LIGO sites are seismically quieter than the campus laboratory by approximately an order of magnitude at relevant frequencies. The dynamic reserve of the controllers can be correspondingly reduced, thereby reducing the torque induced by a given amount of electronic noise in the circuitry.

\section{Conclusions}

Simple geometric considerations can accurately model the sensitivity of Fabry-Perot cavities to highfrequency angular rotations of their mirrors, permitting a straightforward analysis of torque-induced noise. In addition to guiding the design of improved mirror control systems for the LIGO 40-m interferometer, which greatly enhanced the performance of that instrument, the application of the model to planned full-scale observatory interferometers indicates that with available control technology, angular rotations need not compromise LIGO performance at target sensitivity levels.

We thank all those who contributed to the construction and improvement of the interferometer, and we are grateful for the continuing support of LIGO science, engineering, and administrative staff. We are especially grateful to J. Chapsky for designing the improved control electronics and to A. Gillespie, F. Raab, R. Spero, and S. Whitcomb for their assistance in measuring and analyzing the data and preparing the manuscript. Our work is supported by National Science Foundation grant PHY-9210038.

\section{References and Notes}

1. A. Abramovici, W. E. Althouse, R. W. P. Drever, Y. Gürsel, S. Kawamura, F. J. Raab, D. Shoemaker, L. Sievers, R. E. Spero, K. S. Thorne, R. E. Vogt, R. Weiss, S. E. Whitcomb, and M. E. Zucker, "LIGO: the laser interferometer gravitational wave observatory," Science 256, 325-333 (1992).

2. R. E. Vogt, "The U.S. LIGO project," in Proceedings of the Sixth Marcel Grossmann Meeting on General Relativity, $\mathrm{H}$. Sato and T. Nakamura, eds. (World Scientific, Singapore, 1991), p. 244.

3. S. L. Smith, "A search for gravitational waves from coalescing binary stars using the Caltech 40 meter gravity wave detector," Ph.D. dissertation (California Institute of Technology, Pasadena, Calif., 1988), p. 37.

4. Equation (1) and the rest of the discussion can be readily applied to any cavity geometry by the suitable redefinition of $\alpha, \beta$, and $\gamma$. In general, $\beta$ and $\gamma$ are not linearly dependent.

5. R. G. Brown, Introduction to Random Signal Analysis and Kalman Filtering (Wiley, New York, 1983), Chap. 2, p. 85.

6. S. O. Rice, "Mathematical analysis of random noise," in Selected Papers on Noise and Stochastic Processes, N. Wax, ed. (Dover, New York, 1954), pp. 133-294.

7. M. E. Zucker, "The LIGO $40 \mathrm{~m}$ prototype laser interferometer gravitational wave detector," in Proceedings of the Sixth Marcel Grossmann Meeting on General Relativity, H. Sato and T. Nakamura, eds. (World Scientific, Singapore, 1991), p. 224.

8. S. Kawamura, "Test mass orientation noise in the LIGO $40 \mathrm{~m}$ 
prototype," in Proceedings of the Sixth Marcel Grossmann Meeting on General Relativity, H. Sato and T. Nakamura, eds. (World Scientific, Singapore, 1991), p. 1486.

9. Remote LIGO sites exhibit approximately one tenth the seismic amplitude found in the campus laboratory at frequencies that determine the rms mirror angle fluctuation. In addition, current seismic isolation stack and suspension designs show one fifth the resonant amplification effect found to enhance rms mirror motion in the $40-\mathrm{m}$ interferometer. Although the hundredfold increase in length would proportionately increase the $d_{\nu}{ }^{\text {rms }}$ arising from a given rms angle fluctuation, these factors should reduce that angle fluctuation by a factor of the order of 50, leading us to expect only a doubling in net $d_{\nu}{ }^{\mathrm{rms}}$ in full-scale interferometers. 